\title{
DIMENSION OF SLICES THROUGH A SELF-SIMILAR SET WITH INITIAL CUBIC PATTERN
}

\author{
Zhixiong Wen, Wen Wu and Lifeng Xi \\ Huazhong University of Science and Technology, School of Mathematics and Statistics \\ 430074, Wuhan, P. R. China; zhi-xiong.wen@mail.hust.edu.cn \\ Huazhong University of Science and Technology, School of Mathematics and Statistics \\ 430074, Wuhan, P. R. China; hust.wuwen@gmail.com \\ Zhejiang Wanli University, Institute of Mathematics \\ 315100, Ningbo, P.R. China; xilifengningbo@yahoo.com
}

\begin{abstract}
In this paper, we give a sufficient condition to ensure that the typical Hausdorff dimension of slices through a self-similar set in a fixed direction takes the value in Marstrand's theorem, i.e., the dimension of the self-similar set minus one.
\end{abstract}

\section{Introduction}

The intersections of Borel sets in $\mathbf{R}^{n}$ with $(n-m)$-dimensional subspaces in random directions are studied in many publications. The following Marstrand's theorem [8] (also see [9] Chapter 10) is well known: suppose $A \subset \mathbf{R}^{n}$ is a Borel set with $0<\mathcal{H}^{s}(A)<\infty$ and $m<s<n$, then for $\gamma_{n, n-m}$-almost all $(n-m)$-dimensional subspace $V$ and $\mathcal{H}^{s}$-almost all $x \in A$,

$$
\operatorname{dim}_{H}[A \cap(V+x)]=s-m .
$$

This theorem was first proved by Marstrand [8] for the intersection of planar sets with lines. Later, Mattila [9] proved its higher-dimensional version. In particular, when $m=1$, we call $(s-1)$ the Marstrand's value of Hausdorff dimension for the slices.

Wen and $\mathrm{Xi}$ [11] studied the slices of scaling self-similar set $E=\cup_{i}\left(r_{i} E+b_{i}\right)$ $\left(r_{i} \in(0,1)\right)$ in $\mathbf{R}^{n}$, and obtained that for a fixed $(n-m)$-dimensional subspace $V$, $\operatorname{dim}_{H}[E \cap(V+a)]$ is constant for $\mathcal{H}^{m}$-almost all $a \in V^{\perp}$ with $E \cap(V+a) \neq \emptyset$.

Kenyon and Peres proved in [5] that given two Cantor set $X$ and $Y$ in $[0,1)$, invariant under the map $x \mapsto b x(\bmod 1)$, the Hausdorff dimension of $(X+t) \cap Y$ is constant almost everywhere.

The following results showed that for some special planar sets, their typical dimension of the slices in a fixed direction are strictly less than the Marstrand's value. Let $\mathcal{L}$ denote the Lebesgue measure on $\mathbf{R}$.

- Hawkes obtained in [3] that

doi:10.5186/aasfm.2013.3836

2010 Mathematics Subject Classification: Primary 28A80.

Key words: Self-similar set, slice, Hausdorff dimension, box dimension.

The work is supported by NSFC (Grant Nos. 11071224, 11071090), Program for New Century Excellent Talents in University of China and NSF of Zhejiang Province (No. LY12F02011).

Lifeng $\mathrm{Xi}$ is the corresponding author. 


$$
\operatorname{dim}_{H}[(C \times C) \cap\{(x, y): y=x+b\}]=\frac{\log 2}{3 \log 3}<\operatorname{dim}_{H}[C \times C]-1
$$

for $\mathcal{L}$-almost all $b \in[-1,1]$.

- Benjamini and Peres [2] gave a class of fractal sets in the unit square $[0,1] \times$ $[0,1]$ with $\operatorname{dim}_{H} F=\frac{\log 3}{\log 2}$ and proved that for any $F$ in this class,

$$
\operatorname{dim}_{H}\{y \in[0,1]:(x, y) \in F\} \geq 1 / 2
$$

for $\mathcal{L}$-almost all $x \in[0,1]$.

- Liu, Xi and Zhao [6] investigated the Sierpinski carpet with $\operatorname{dim}_{H} E=\frac{\log 8}{\log 3}$. Denote by $L_{k, b}$ the line $y=k x+b$. For any fixed $k \in \mathbf{Q}^{+}$, they proved that

$$
\operatorname{dim}_{H}\left[E \cap L_{k, b}\right]=\operatorname{dim}_{B}\left[E \cap L_{k, b}\right]=c_{k}
$$

for $\mathcal{L}$-almost all $b \in[-k, 1]$, where the constant $c_{k}$ depends only on the rational slope $k$ and

$$
c_{k} \leq \log 8 / \log 3-1=\operatorname{dim}_{H} E-1 .
$$

They posed a conjecture that $c_{k}<\log 8 / \log 3-1$.

- Manning and Simon [7] proved the conjecture: for any fixed $k \in \mathbf{Q}^{+}$,

$$
\operatorname{dim}_{H}\left[E \cap L_{k, b}\right]=\operatorname{dim}_{B}\left[E \cap L_{k, b}\right]=c_{k}<\operatorname{dim}_{H} E-1
$$

for $\mathcal{L}$-almost all $b \in[-k, 1]$.

- Bárány, Ferguson and Simon [1] studied the Sierpinski gasket in $\mathbf{R}^{2}$. Let $E_{\theta, b}=E \cap\{(x, y): y=\tan \theta \cdot x+b\}$, where $\theta \in\left(0, \frac{\pi}{3}\right)$ and $\tan \theta=\frac{\sqrt{3} p}{2 q+p}$ with $p, q \in \mathbf{N}$. Then there exists constant $\alpha(\theta)$ depending only on $\theta$ such that for $\mathcal{L}$-almost all $b \in \Delta_{\theta}$,

$$
\operatorname{dim}_{H} E_{\theta, b}=\operatorname{dim}_{B} E_{\theta, b}=\alpha(\theta)<\operatorname{dim}_{H} E-1 .
$$

In the above literatures, the typical dimensions of the corresponding slices do not take the Marstrand's value. Naturally, we will ask: when slicing the self-similar set, can we ensure that the typical dimension of the slices takes the Marstrand's value?

$\mathrm{Wu}$ and $\mathrm{Xi}[12]$ discussed the intersections of a class of generalized Sierpinski sponges in $\mathbf{R}^{n}$ with an $(n-1)$-dimensional hyperplane of the form

$$
\left\{x \in \mathbf{R}^{n}: a \cdot x=b\right\}
$$

where $a \in \mathbf{Z}^{n} \backslash\{0\}$ and $b \in \mathbf{Z}$. They give a sufficient condition, under which the Hausdorff dimensions of slices takes the Marstrand's value.

In this paper, we discuss the slices for $b \in \mathbf{R}$. When $b \in \mathbf{Z}$, after certain modification the slices satisfy the graph-directed construction. When $b \in \mathbf{R}$, the nested structure, which the slices have, is different to the graph-directed construction. Thus we use the method in [5] and [6], and show that the typical Hausdorff dimension of slices in a suitable fixed rational direction takes the Marstrand's value.

Let $m \geq 2$ be an integer and $\Omega \subset\{0,1, \cdots,(m-1)\}^{n}(n \geq 2)$. For all $v \in \Omega$, let $f_{v}(x)=(x+v) / m$. The self-similar set

$$
E=\bigcup_{v \in \Omega} f_{v}(E)=\bigcup_{v \in \Omega} \frac{1}{m}(E+v)
$$

is called a self-similar set with initial cubic pattern $\left\{\frac{1}{m}\left([0,1]^{n}+v\right)\right\}_{v \in \Omega}$. Then $\operatorname{dim}_{H} E=$ $\log (\# \Omega) / \log m$ (see e.g. [4]). 
Fix $a=\left(a_{1}, a_{2}, \cdots, a_{n}\right) \in \mathbf{Z}^{n} \backslash\{0\}$. For $b \in \mathbf{R}$, let $\Pi_{a, b}$ be the hyperplane in $\mathbf{R}^{n}$ defined by

$$
\Pi_{a, b}=\left\{x \in \mathbf{R}^{n}: a \cdot x=b\right\},
$$

and the slice $E_{a, b}=E \cap \prod_{a, b}$.

Let $\mathbf{T}^{n}$ be the $n$-dimensional torus and $P: \mathbf{R}^{n} \rightarrow \mathbf{T}^{n}$ the map defined by $P\left(x_{1}, \cdots, x_{n}\right)=\left(y_{1}, \cdots, y_{n}\right) \in \mathbf{T}^{n}$ where $y_{i}=\left\{x_{i}\right\}$ the fractional part of $x_{i}$ for every $i$. Set $F=P(E)$, then $F$ is an invariant set with respect to the expansive mapping $\tau(y)=m y$. For $b \in \mathbf{R}$, write

$$
F_{a, b}=F \cap\left\{y=\left(y_{1}, \cdots, y_{n}\right) \in \mathbf{T}^{n}: \sum_{i=1}^{n} a_{i} y_{i} \equiv b(\bmod 1)\right\} .
$$

The main results of the paper are stated as follows.

Theorem 1. If there exists a positive integer $s$ such that

$$
\#\{v \in \Omega: a \cdot v \equiv t(\bmod m)\}=s
$$

for all $t \in\{0,1, \cdots, m-1\}$, then

(1) for all $b \in \mathbf{R}$,

$$
\operatorname{dim}_{B} F_{a, b}=\operatorname{dim}_{B}\left(\bigcup_{i \in \mathbf{Z}} E_{a, b+i}\right)=\frac{\log s}{\log m}=\operatorname{dim}_{H} E-1
$$

(2) for $\mathcal{L}$-a.e. $b \in \mathbf{R}$,

$$
\operatorname{dim}_{H} F_{a, b}=\operatorname{dim}_{H}\left(\bigcup_{i \in \mathbf{Z}} E_{a, b+i}\right)=\frac{\log s}{\log m}=\operatorname{dim}_{H} E-1 .
$$

Remark 1. Under the assumption $(\star)$, Theorem 1 in [12] showed that for $b \in \mathbf{Z}$,

$$
\operatorname{dim}_{B}\left(\cup_{i \in \mathbf{Z}} E_{a, b+i}\right)=\operatorname{dim}_{H}\left(\cup_{i \in \mathbf{Z}} E_{a, b+i}\right)=\operatorname{dim}_{H} E-1 .
$$

When $b \in \mathbf{R} \backslash \mathbf{Z}$, it is not clear that whether the Hausdorff dimension of slices $\cup_{i \in \mathbf{Z}} E_{a, b+i}$ equals to its box dimension or not.

Remark 2. For $b \in \mathbf{Z}$, the slices have the graph-directed construction (see [12]). To compute the box dimension, we only need to evaluate the spectral radius of a fixed adjacency matrix. When $b$ is not an integer, the slices fail to have the graph-directed construction. To evaluate the box dimension, we need to compute the Lyapunov exponent $\lim _{k \rightarrow \infty} \frac{\log \left\|M(b) M(m b) \cdots M\left(m^{k} b\right)\right\|}{k}$, where the matrix $M(t)$ is variable for $t$ (see Section 3).

Let $\Lambda:=\left\{b \in \mathbf{R}: E \cap \Pi_{a, b} \neq \emptyset\right\}$. Then $\Lambda$ is self-similar. We have $\mathcal{L}(\Lambda) \geq 1$ under the assumption $(\star)$ (see Proposition 4 ).

Theorem 2. Preserve the assumption of Theorem 1. Then for $\mathcal{L}$-a.e. $b \in \Lambda$,

$$
\operatorname{dim}_{H} E_{a, b}=\operatorname{dim}_{B} E_{a, b}=\frac{\log s}{\log m}=\operatorname{dim}_{H} E-1 .
$$

Remark 3. Under the assumption $(\star)$, Theorem 2 answer the question on typical dimension of slices.

For $\Omega \subset\{0,1, \cdots, m-1\}^{n}, n \geq 2$, then the self-similar set $E=\bigcup_{v \in \Omega} \frac{E+v}{m}$ has Hausdorff dimension $\operatorname{dim}_{H} E=\frac{\log \# \Omega}{\log m}$. Then $\operatorname{dim}_{H} E>1$ if and only if

$$
\# \Omega>m \text {. }
$$


From Theorem 2 and the results listed above, we pose the following conjecture.

Conjecture 1. Suppose $E=\bigcup_{v \in \Omega} \frac{E+v}{m}$ with $\# \Omega>m$. Let $a \in \mathbf{Z}^{n} \backslash\{0\}$ and $d_{a}$ the typical value of $\operatorname{dim}_{H} E_{a, b}$ for $\mathcal{L}$-almost all $b \in \Lambda$. Then

$$
d_{a}=\operatorname{dim}_{H} E-1
$$

if and only if the assumption $(\star)$ holds for $\Omega$.

In this paper, we face the following difficulty: in the Sierpinski carpet, we have

$$
\Pi_{a, b} \cap E \neq \emptyset \text { iff } \Pi_{a, b} \cap[0,1]^{n} \neq \emptyset,
$$

note that $[0,1]^{n} \cap \Pi_{a, b} \neq \emptyset$ iff $b \in\left[A^{-}, A^{+}\right]$, where

$$
A^{-}=\sum_{a_{i}<0} a_{i}, A^{+}=\sum_{a_{i}>0} a_{i}
$$

and set $A^{-}=0$ if $\left\{i: a_{i}<0\right\}=\emptyset$ and $A^{+}=0$ if $\left\{i: a_{i}>0\right\}=\emptyset$. Thus we obtain that

$$
\Pi_{a, b} \cap E \neq \emptyset \quad \text { iff } \quad b \in\left[A^{-}, A^{+}\right]
$$

whereas for the self-similar set with initial cubic pattern, the property (1.2) does not hold as in the following example and thus the claim (1.3) also fails. That means we do not know when the slice $E \cap \Pi_{a, b} \neq \emptyset$. We overcome this difficulty by introducing an equivalent condition ([12, Lemma 1] or Lemma 6 in Section 3) to determine that when the slice is not empty.

Example 1. Let $a=(3,-4)$ and $\Pi_{a, b}$ the line $3 x-4 y=b$.

(1) When $m=3$ and $\Omega=\{0,1,2\}^{2} \backslash\{(1,1)\}, E$ is the Sierpinski carpet and $E \cap \Pi_{a, b} \neq \emptyset$ if $\Pi_{a, b}$ touches $[0,1]^{2}$ (Fig. 1).

(2) When $m=4$ and $\Omega=\{(0,1),(0,2),(1,2),(1,3),(2,0),(2,3),(3,0),(3,1)\}, E$ is a self-similar set with initial cubic pattern. In this case, the assumption $(\star)$ holds and $E \cap\{(x, y): 3 x-y=0\}=\emptyset$ (Fig. 2).

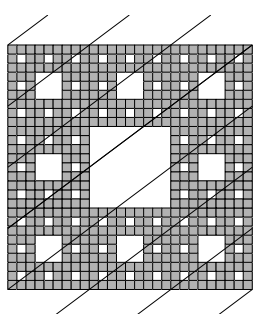

Figure 1. Slices of Sierpinski carpet.

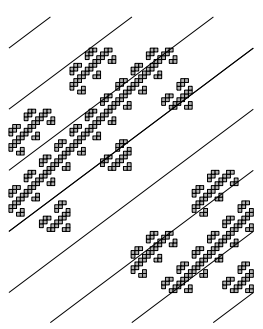

Figure 2. The case that empty slices occur.

The rest of the paper is organized as follows. In Section 2, we give some basic results on discrete structure $\{b+i: i \in \mathbf{Z}\} \cap\left[A^{-}, A^{+}\right]$and images of slices by map $P$. In Section 3, we investigate the box dimensions of $F_{a, b}$ and $\bigcup_{i \in \mathbf{Z}} E_{a, b+i}$ to prove Theorem 1(1). Section 4 is devoted to Theorem 1(2) on Hausdorff dimension. In Section 5, we discuss the Hausdorff and box dimension of $E_{a, b}$ for $b \in \Lambda$ and prove Theorem 2. 


\section{Preliminaries}

Fix $\Omega \subset\{0,1, \cdots, m-1\}^{n}$ and

$$
a=\left(a_{1}, \cdots, a_{n}\right) \in \mathbf{Z}^{n} \backslash\{0\}
$$

satisfying for every $t \in\{0,1, \cdots,(m-1)\}$,

$$
\#\{v \in \Omega: a \cdot v \equiv t(\bmod m)\}=s .
$$

For all $v \in \Omega$, let

$$
f_{v}(x)=(x+v) / m .
$$

Let $\Pi_{a, b}(b \in \mathbf{R})$ be the hyperplane in $\mathbf{R}^{n}$ defined by

$$
\Pi_{a, b}=\left\{x \in \mathbf{R}^{n}: a \cdot x=b\right\},
$$

and the slice $E_{a, b}=E \cap \Pi_{a, b}$, the intersection of the self-similar set with the hyperplane. For any $v \in \Omega$, set

$$
T_{v}(x)=m x-a \cdot v, \quad S_{v}(x)=T_{v}^{-1}(x)=(x+a \cdot v) / m .
$$

Note that

$$
f_{v}^{-1}\left(\Pi_{a, b}\right)=\Pi_{a, T_{v}(b)} .
$$

Using the above formula, we can check that

$$
f_{v_{1} \cdots v_{k}}(E) \cap \Pi_{a, z}=f_{v_{1} \cdots v_{k}}\left(E \cap \prod_{a, T_{v_{k} \cdots v_{1}}(z)}\right) .
$$

Recall that

$$
A^{-}=\sum_{a_{i}<0} a_{i}, \quad A^{+}=\sum_{a_{i}>0} a_{i},
$$

and set $A^{-}=0$ if $\left\{i: a_{i}<0\right\}=\emptyset$ and $A^{+}=0$ if $\left\{i: a_{i}>0\right\}=\emptyset$. Then $A^{+}-A^{-}=$ $\|a\|_{1}$, where the norm of a row vector is given by

$$
\left\|\left(x_{1}, \cdots, x_{n}\right)\right\|_{1}=\sum_{i=1}^{n}\left|x_{i}\right| .
$$

It is easy to see that

$$
[0,1]^{n} \cap \Pi_{a, b} \neq \emptyset \quad \text { if and only if } b \in\left[A^{-}, A^{+}\right] .
$$

We also need the following lemma, for a proof, refer to [12, equation (1.4)].

Lemma 1. [12] For any $v \in \Omega$,

$$
\begin{array}{r}
S_{v}\left(\left[A^{-}, A^{+}\right]\right) \subset\left[A^{-}, A^{+}\right], \\
T_{v}\left(\left[A^{-}, A^{+}\right]^{c}\right) \subset\left[A^{-}, A^{+}\right]^{c} .
\end{array}
$$

2.1. Discrete Structure $\boldsymbol{\Gamma}_{\boldsymbol{b}}$. Let $D=\left\{b \in \mathbf{R}: m^{k} b \notin \mathbf{Z}\right.$ for any integer $k \geq$ $0\}$. Let $\tau_{0}: \mathbf{R} \rightarrow \mathbf{R}$ be the map $\tau_{0}(x)=m x$. It is easy to see that for any integer $k \geq 0$,

$$
\tau_{0}^{k}(D) \subset D \quad \text { and } \quad \tau_{0}^{k}\left(D^{c}\right) \subset D^{c} .
$$

For any $b \in \mathbf{R}$, let

$$
\Gamma_{b}=\left\{b+i \in\left[A^{-}, A^{+}\right]: i \in \mathbf{Z}\right\} \subset\left[A^{-}, A^{+}\right] .
$$


Then $\Gamma_{b_{1}}=\Gamma_{b_{2}}$ if $b_{1} \equiv b_{2}(\bmod 1)$ and

$$
\# \Gamma_{b}= \begin{cases}A^{+}-A^{-}+1, & \text { if } b \in \mathbf{Z} \\ A^{+}-A^{-}, & \text {otherwise. }\end{cases}
$$

In particular,

$$
\# \Gamma_{m^{k} b}=A^{+}-A^{-} \quad \text { for any } b \in D \text { and } k \geq 0 \text {, }
$$

and

$$
\Gamma_{b} \equiv\left\{A^{-},\left(A^{-}+1\right), \cdots, A^{+}\right\} \quad \text { for all } b \in \mathbf{Z} .
$$

Given $b \in \mathbf{R}$, we arrange the element of $\Gamma_{b}$ in ascending order, i.e.,

$$
\Gamma_{b}(1)<\Gamma_{b}(2)<\cdots<\Gamma_{b}\left(\# \Gamma_{b}\right) .
$$

Set

$$
I_{i}:=\left[A^{-}-1+i, A^{-}+i\right)\left(1 \leq i \leq A^{+}-A^{-}\right) .
$$

When $i \leq A^{+}-A^{-}$, we have

$$
\Gamma_{b}(i) \in I_{i} \quad \text { for all } b \in \mathbf{R} .
$$

Whereas $i=A^{+}-A^{-}+1$ we have

$$
\Gamma_{b}\left(A^{+}-A^{-}+1\right)=A^{+} \text {for all } b \in \mathbf{Z} .
$$

Since $a \cdot v \in \mathbf{Z}$, we obtain the following lemma.

Lemma 2. If $z \in \Gamma_{b}$ and $T_{v}(z) \in\left[A^{-}, A^{+}\right]$, then $T_{v}(z) \in \Gamma_{m b}$.

2.2. Projection from $\mathrm{R}^{n}$ to $\mathrm{T}^{n}$. Let

$$
\mathbf{T}^{n}=\mathbf{R}^{n} / \mathbf{Z}^{n}
$$

be the $n$-dimensional torus and $P$ the corresponding natural mapping from $\mathbf{R}^{n}$ to $\mathbf{T}^{n}$. For $y, y^{\prime} \in \mathbf{T}^{n}$, the metric $d$ on $\mathbf{T}^{n}$ is defined as follow

$$
d\left(y, y^{\prime}\right)=\left(\sum_{i=1}^{n}\left(\min \left\{\left|y_{i}-y_{i}^{\prime}\right|, 1-\left|y_{i}-y_{i}^{\prime}\right|\right\}\right)^{2}\right)^{1 / 2}=\min _{\substack{P(x)=y \\ P\left(x^{\prime}\right)=y^{\prime}}}\left|x-x^{\prime}\right| .
$$

Now, we will map the slice $E_{a, b}$ into $\mathbf{T}^{n}$. Let $\tau: \mathbf{T}^{n} \rightarrow \mathbf{T}^{n}$ be the map $\tau(y)=m y$. Set

$$
K=P\left(\bigcup_{v \in \Omega} f_{v}\left([0,1]^{n}\right)\right) \subset \mathbf{T}^{n} .
$$

Suppose $F=\left\{y \in \mathbf{T}^{n}: \tau^{k}(y) \in K, \forall k \geq 0\right\}$, then

$$
F=P(E)
$$

where $E$ is the self-similar set defined by (1.1).

Recall that $a=\left(a_{1}, \cdots, a_{n}\right) \in \mathbf{Z}^{n} \backslash\{0\}$. For $b \in \mathbf{R}$, let

$$
F_{a, b}=F \cap\left\{y=\left(y_{1}, \cdots, y_{n}\right) \in \mathbf{T}^{n}: \sum_{i=1}^{n} a_{i} y_{i} \equiv b(\bmod 1)\right\} .
$$

Lemma 3. For any $b \in \mathbf{R}$, we have

$$
P\left(\bigcup_{z \in \Gamma_{b}} E_{a, z}\right)=F_{a, b}
$$


Proof. For any $x \in \bigcup_{z \in \Gamma_{b}} E_{a, z}$, we have $\sum_{i=1}^{n} a_{i} x_{i} \equiv b(\bmod 1)$. Suppose $y=$ $P(x)$, then $y \in F$ and $\sum_{i=1}^{n} a_{i} y_{i} \equiv b(\bmod 1)$. Hence

$$
P\left(\bigcup_{z \in \Gamma_{b}} E_{a, z}\right) \subset F_{a, b} .
$$

On the other hand, suppose $y \in F_{a, b}$ with $\sum_{i=1}^{n} a_{i} y_{i} \equiv b(\bmod 1)$. Since $P(E)=$ $F$, there exists $x \in E$ such that $P(x)=y$. Then $\sum_{i=1}^{n} a_{i} x_{i}=z \in\{b+i: i \in \mathbf{Z}\}$. Note that $x \in E \subset[0,1]^{n}$, we have $\Pi_{a, z} \cap[0,1]^{n} \neq \emptyset$, which implies $z \in\left[A^{-}, A^{+}\right]$by (2.3), i.e., $z \in \Gamma_{b}=\left\{b+i \in\left[A^{-}, A^{+}\right]: i \in \mathbf{Z}\right\}$. Hence $y=P(x)$, where $x \in E \cap \Pi_{a, z}=E_{a, z}$ with $z \in \Gamma_{b}$. Therefore

$$
F_{a, b} \subset P\left(\bigcup_{z \in \Gamma_{b}} E_{a, z}\right) .
$$

Notice that $E \subset[0,1]^{n}$ and there exists a constant $\delta>0$ such that

$$
d\left(P(x), P\left(x^{\prime}\right)\right)=\left|x-x^{\prime}\right|
$$

whenever $\left|x-x^{\prime}\right|<\delta$. Therefore, we have the following result.

Lemma 4. For any $b \in \mathbf{R}$, we have

$$
\operatorname{dim} \bigcup_{z \in \Gamma_{b}} E_{a, z}=\operatorname{dim} F_{a, b}
$$

where 'dim' stands for any one of $\operatorname{dim}_{H}, \underline{\operatorname{dim}}_{B}$ and $\overline{\operatorname{dim}}_{B} F_{a, b}$.

\section{Box dimension of sections}

The nested structure of the slices $\left\{E_{a, b}\right\}_{b \in\left[A^{-}, A^{+}\right]}$is characterized in the following Lemma, which is similar to Proposition 1 in [12].

Lemma 5. For any $b \in\left[A^{-}, A^{+}\right]$,

$$
E_{a, b}=\bigcup_{v \in \Omega} f_{v}\left(E_{a, T_{v}(b)}\right)=\bigcup_{v \in \Omega} \frac{E_{a, T_{v}(b)}+v}{m} .
$$

Further, for $k \geq 1$,

$$
\begin{aligned}
E \cap \Pi_{a, b} & =\bigcup_{v_{1} \cdots v_{k} \in \Omega^{k}} f_{v_{1} \cdots v_{k}}\left(E \cap \Pi_{a, T_{v_{k} \cdots v_{1}}(b)}\right) \\
& =\bigcup_{T_{v_{k} \cdots v_{1}}(b) \in \Gamma_{m^{k}} b} f_{v_{1} \cdots v_{k}}\left(E \cap \Pi_{a, T_{v_{k} \cdots v_{1}}(b)}\right) .
\end{aligned}
$$

Proof. The equation (3.1) follows from Proposition 1 in [12].

For (3.2), using induction, we have

$$
E \cap \Pi_{a, b}=\bigcup_{v_{1} \cdots v_{k} \in \Omega^{k}} f_{v_{1} \cdots v_{k}}\left(E \cap \Pi_{a, T_{v_{k} \cdots v_{1}}(b)}\right) .
$$

Further, if $T_{v_{k} \cdots v_{1}}(b) \in\left[A^{-}, A^{+}\right]^{c}$, then $[0,1]^{n} \cap \Pi_{a, T_{v_{k} \cdots v_{1}}(b)}=\emptyset$ by $(2.3)$, and thus

$$
E \cap \Pi_{a, T_{v_{k} \cdots v_{1}}(b)}=\emptyset .
$$

Combine this fact and Lemma 2, we have

$$
E \cap \Pi_{a, b}=\bigcup_{T_{v_{k} \cdots v_{1}}(b) \in \Gamma_{m^{k} b}} f_{v_{1} \cdots v_{k}}\left(E \cap \Pi_{a, T_{v_{k} \cdots v_{1}}(b)}\right) .
$$


Remark 4. Some $E_{a, T_{v}(b)}$ in (3.1) may be empty.

The following lemma describes when the intersection $E \cap \Pi_{a, b}$ is not empty. This has been done in [12] for integer $b \in\left[A^{-}, A^{+}\right]$. In fact the proof in [12] holds for all $b \in\left[A^{-}, A^{+}\right]$.

Lemma 6. [12, Lemma 1] For any $b \in\left[A^{-}, A^{+}\right]$,

$E \cap \Pi_{a, b} \neq \emptyset \Longleftrightarrow \exists v_{1} \cdots v_{k} \cdots \in \Omega^{\infty}$, s.t. $T_{v_{k} \cdots v_{1}}(b) \in\left[A^{-}, A^{+}\right]$for all $k>0$.

Lemma 5 and Lemma 2 implies the following fact.

Claim 1. For any $z \in \Gamma_{b}, E_{z}$ is composed of some reduced (with ratio $1 / m$ ) copies of $E_{z^{\prime}}$ for some $z^{\prime} \in \Gamma_{m b}$.

We record the number of copies with a non-negative integer matrix.

For $b \in \mathbf{R}$, the integer matrix $M(b)=\left(c_{i, j}\right)_{1 \leq i \leq \# \Gamma_{b}, 1 \leq j \leq \# \Gamma_{m b}}$ is defined by

$$
c_{i, j}=\#\left\{v \in \Omega: T_{v}\left(\Gamma_{b}(i)\right)=\Gamma_{m b}(j)\right\},
$$

where $c_{i, j}$ is the number of reduced copies of $E_{\Gamma_{m b}(j)}$ contained in $E_{\Gamma_{b}(i)}$.

- If $b \in D$, then $M(b)$ is an $\left(A^{+}-A^{-}\right) \times\left(A^{+}-A^{-}\right)$matrix.

- If $b \in \mathbf{Z}$, then $M(b)$ is an $\left(A^{+}-A^{-}+1\right) \times\left(A^{+}-A^{-}+1\right)$ matrix.

- For $b \in D^{c} \backslash \mathbf{Z}$, suppose $m^{k} b \in \mathbf{Z}$ and $m^{k-1} b \notin \mathbf{Z}$, then $M\left(m^{k-1} b\right)$ is an $\left(A^{+}-A^{-}\right) \times\left(A^{+}-A^{-}+1\right)$ matrix and $M\left(m^{t} b\right)$ is a square matrix for any non-negative integer $t \neq k-1$.

Let $\mathbf{1}_{b}=(1, \cdots, 1)$ be a vector in $\mathbf{R}^{\# \Gamma_{b}}$ with every coordinate 1 . i.e.,

$$
\mathbf{1}_{b} M(b)=s \mathbf{1}_{m b} .
$$

Proof. For all $1 \leq j \leq \# \Gamma_{m b}$, we need to show that

$$
\sum_{i=1}^{\# \Gamma_{b}} c_{i, j}=s .
$$

By (3.3), we have

$$
\begin{aligned}
\sum_{i=1}^{\# \Gamma_{b}} c_{i, j} & =\sum_{i=1}^{\# \Gamma_{b}} \#\left\{v \in \Omega: T_{v}\left(\Gamma_{b}(i)\right)=\Gamma_{m b}(j)\right\} \\
& =\#\left\{v \in \Omega: \exists z \in \Gamma_{b}, \text { s.t. } T_{v}(z)=\Gamma_{m b}(j)\right\} .
\end{aligned}
$$

Suppose that $z=b+i^{\prime}, \Gamma_{m b}(j)=m b+j^{\prime}$ where $i^{\prime}, j^{\prime} \in \mathbf{Z}$. Then

$$
\begin{aligned}
T_{v}(z)=\Gamma_{m b}(j) & \Longrightarrow \\
& \Longrightarrow a \cdot v b+m i^{\prime}-a \cdot v=m b+j^{\prime}(\bmod m) .
\end{aligned}
$$

Hence

$s=\#\left\{v \in \Omega: a \cdot v \equiv-j^{\prime}(\bmod m)\right\} \geq \#\left\{v \in \Omega: \exists z \in \Gamma_{b}\right.$, s.t. $\left.T_{v}(z)=\Gamma_{m b}(j)\right\}$.

Conversely, for any $v \in \Omega$ satisfying $a \cdot v \equiv-j^{\prime}(\bmod m)$, we have

$$
S_{v}\left(\Gamma_{m b}(j)\right)=S_{v}\left(m b+j^{\prime}\right)=b+\frac{j^{\prime}+a \cdot v}{m} \in b+\mathbf{Z} .
$$


By (2.4), we know that $S_{v}\left(\Gamma_{m b}(j)\right) \in\left[A^{-}, A^{+}\right]$. That means

$$
S_{v}\left(\Gamma_{m b}(j)\right) \in \Gamma_{b},
$$

i.e., $\exists z \in \Gamma_{b}$, s.t. $T_{v}(z)=\Gamma_{m b}(j)$. Therefore

$$
s=\#\left\{v \in \Omega: a \cdot v \equiv-j^{\prime}(\bmod m)\right\} \leq \#\left\{v \in \Omega: \exists z \in \Gamma_{a} \text {, s.t. } T_{v}(z)=\Gamma_{m b}(j)\right\} .
$$

This completes the proof.

Write $f_{v_{1} \cdots v_{k}}=f_{v_{1}} \circ \cdots \circ f_{v_{k}}$. Let

$$
N_{k}(b)=\sum_{z \in \Gamma_{b}} \#\left\{v_{1} \cdots v_{k} \in \Omega^{k}: f_{v_{1} \cdots v_{k}}\left([0,1]^{n}\right) \cap \Pi_{a, z} \neq \emptyset\right\} .
$$

We also have

$$
N_{k}(b)=\sum_{z \in \Gamma_{b}} \#\left\{v_{1} \cdots v_{k} \in \Omega^{k}: T_{v_{k} \cdots v_{1}}(z) \in\left[A^{-}, A^{+}\right]\right\},
$$

since

$$
\begin{aligned}
f_{v_{1} \cdots v_{k}}\left([0,1]^{n}\right) \cap \Pi_{a, z} \neq \emptyset & \Longleftrightarrow[0,1]^{n} \cap \Pi_{a, T_{v_{k} \cdots v_{1}}(z)} \neq \emptyset \\
& \Longleftrightarrow T_{v_{k} \cdots v_{1}}(z) \in\left[A^{-}, A^{+}\right]
\end{aligned}
$$

due to (2.1) and (2.3).

The following proposition shows us how to compute $N_{k}(b)$.

Lemma 8. For any $k>0$, we have

$$
N_{k}(b)=\left\|\mathbf{1}_{b} M(b) M(m b) \cdots M\left(m^{k-1} b\right)\right\|_{1} .
$$

Proof. First, by (3.6), for any $k>0$, we have

$$
N_{k}(b)=\sum_{z \in \Gamma_{b}} \#\left\{v_{1} \cdots v_{k}: T_{v_{k} \cdots v_{1}}(z) \in \Gamma_{m^{k} b}\right\} .
$$

Now we will show that

$$
\sum_{z \in \Gamma_{b}} \#\left\{v_{1} \cdots v_{k} \in \Omega^{k}: T_{v_{k} \cdots v_{1}}(z) \in \Gamma_{m^{k} b}\right\}=\left\|\mathbf{1}_{b} M(b) M(m b) \cdots M\left(m^{k-1} b\right)\right\|_{1} .
$$

Suppose that $M\left(m^{t} b\right)=\left(c_{i_{t+1} i_{t+2}}\right)_{i_{t+1}, i_{t+2}}$, then we obtain that

$$
\begin{aligned}
& \left\|\mathbf{1}_{b} M(b) M(m b) \cdots M\left(m^{k-1} b\right)\right\|_{1} \\
& =\sum_{i_{1} \cdots i_{k+1}} c_{i_{1} i_{2}} c_{i_{2} i_{3}} \cdots c_{i_{k} i_{k+1}} \\
& =\sum_{i_{1} \cdots i_{k+1}} \prod_{t=1}^{k} \#\left\{v_{t} \in \Omega: T_{v_{t}}\left(\Gamma_{m^{t-1} b}\left(i_{t}\right)\right)=\Gamma_{m^{t} b}\left(i_{t+1}\right)\right\} \\
& =\sum_{i_{1} \cdots i_{k+1}} \#\left\{v_{1} \cdots v_{k} \in \Omega^{k}: T_{v_{t}}\left(\Gamma_{m^{t-1} b}\left(i_{t}\right)\right)=\Gamma_{m^{t} b}\left(i_{t+1}\right) \text { for } 1 \leq t \leq k\right\} \\
& =\sum_{z \in \Gamma_{b}} \#\left\{v_{1} \cdots v_{k} \in \Omega^{k}: T_{v_{1}}(z) \in \Gamma_{m b}, T_{v_{2} v_{1}}(z) \in \Gamma_{m^{2} b}, T_{v_{3} v_{2} v_{1}}(z) \in \Gamma_{m^{3} b},\right. \\
& \left.\quad \cdots, T_{v_{k} \cdots v_{1}}(z) \in \Gamma_{m^{k} b}\right\} \\
& =\sum_{z \in \Gamma_{b}} \#\left\{v_{1} \cdots v_{k}: T_{v_{k} \cdots v_{1}}(z) \in \Gamma_{m^{k} b}\right\}
\end{aligned}
$$


where the last equality follows from (2.5). Therefore

$$
N_{k}(b)=\left\|\mathbf{1}_{b} M(b) M(m b) \cdots M\left(m^{k-1} b\right)\right\|_{1} .
$$

Proposition 1. Every column sum of $M(b) M(m b) \cdots M\left(m^{k-1} b\right)$ is $s^{k}$. Further,

$$
N_{k}(b)=\left(\# \Gamma_{m^{k} b}\right) s^{k} .
$$

Proof. It follows from Lemma 7 that

$$
\mathbf{1}_{b} M(b) M(m b) \cdots M\left(m^{k-1} b\right)=s\left(\mathbf{1}_{m b} M(m b)\right) \cdots M\left(m^{k-1} b\right)=\cdots=s^{k} \mathbf{1}_{m^{k} b} .
$$

By Lemma 8, we have

$$
N_{k}(b)=s^{k}\left\|\mathbf{1}_{m^{k} b}\right\|_{1}=\left(\# \Gamma_{m^{k} b}\right) s^{k} .
$$

Although some $E_{a, b}$ may be empty, the following corollary tells us the union $\bigcup_{z \in \Gamma_{b}} E_{a, z}$ is not empty.

Corollary 1. For any $b \in \mathbf{R}$,

$$
\bigcup_{z \in \Gamma_{b}} E_{a, z} \neq \emptyset
$$

Proof. Suppose on the contrary that $\bigcup_{z \in \Gamma_{b}} E_{a, z}=\emptyset$. Then by Lemma 6 , there exists integer $k$ such that

$$
T_{v_{k} \cdots v_{1}}(z) \notin\left[A^{-}, A^{+}\right] \text {for all } z \in \Gamma_{b} \text { and } v_{1} \cdots v_{k} \in \Omega^{k} .
$$

It follows from (3.6) that

$$
N_{k}(b)=0 .
$$

However, by Proposition $1, N_{k}(b)=\left(\# \Gamma_{m^{k} b}\right) s^{k} \geq\left(A^{+}-A^{-}\right) s^{k}>0$. It is a contradiction.

Let

$$
V_{k}(b)=\sum_{z \in \Gamma_{b}} \#\left\{v_{1} \cdots v_{k} \in \Omega^{k}: f_{v_{1} \cdots v_{k}}(E) \cap \Pi_{a, z} \neq \emptyset\right\} .
$$

Then we have the following estimate.

Corollary 2. For any $b \in \mathbf{R}$ and $k>0$,

$$
V_{k}(b) \geq s^{k} \text {. }
$$

Proof. The last corollary implies there exists $z \in \Gamma_{b}$ such that $E \cap \Pi_{a, z} \neq \emptyset$. In the same way, there exists $z^{*} \in \Gamma_{m^{k} b}$ such that $E \cap \Pi_{a, z^{*}} \neq \emptyset$.

By Lemma 5, we obtain that

$$
\begin{aligned}
\bigcup_{z \in \Gamma_{b}} E \cap \Pi_{a, z} & =\bigcup_{z \in \Gamma_{b}} \bigcup_{v_{1} \cdots v_{k} \in \Omega^{k}} f_{v_{1} \cdots v_{k}}\left(E \cap \Pi_{a, T_{v_{k} \cdots v_{1}}(z)}\right) \\
& =\bigcup_{z^{\prime} \in \Gamma_{m^{k}} b} \bigcup_{z \in \Gamma_{b}} \bigcup_{T_{v_{k} \cdots v_{1}}(z)=z^{\prime}} f_{v_{1} \cdots v_{k}}\left(E \cap \Pi_{a, z^{\prime}}\right) .
\end{aligned}
$$

Then

$$
f_{v_{1} \cdots v_{k}}\left(E \cap \Pi_{a, z^{*}}\right)=f_{v_{1} \cdots v_{k}}(E) \cap \Pi_{a, z} \neq \emptyset
$$

for any $T_{v_{k} \cdots v_{1}}(z)=z^{*}$ with $z \in \Gamma_{b}$. Denote

$$
\alpha_{k}=\#\left\{v_{1} \cdots v_{k}: f_{v_{1} \cdots v_{k}}\left(E \cap \Pi_{a, z^{*}}\right) \neq \emptyset \text { with } T_{v_{k} \cdots v_{1}}(z)=z^{*} \text { for some } z \in \Gamma_{b}\right\} .
$$

Hence

$$
V_{k}(b) \geq \alpha_{k} .
$$


For $M(b) M(m b) \cdots M\left(m^{k-1} b\right)$, when we consider its column sum with respect to $z^{*}$, by Proposition 1 we have

which implies $V_{k}(b) \geq s^{k}$.

$$
\alpha_{k} \geq s^{k}
$$

Consider the union $\bigcup_{z \in \Gamma_{b}}\left[E \cap \Pi_{a, z}\right]$ of slices, we have

Proposition 2. For all $b \in \mathbf{R}$,

$$
\operatorname{dim}_{B}\left(\bigcup_{z \in \Gamma_{b}}\left[E \cap \Pi_{a, z}\right]\right)=\frac{\log s}{\log m}=\operatorname{dim}_{H} E-1 .
$$

Proof. Let $U_{k}(b)$ be the number of $m$-adic squares of side length $m^{-k}$ intersecting $\bigcup_{z \in \Gamma_{b}}\left[E \cap \Pi_{a, z}\right]$. By the definition of the box dimension, we have

$$
\begin{aligned}
& \overline{\operatorname{dim}}_{B}\left(\bigcup_{z \in \Gamma_{b}}\left[E \cap \Pi_{a, z}\right]\right)=\limsup _{k \rightarrow \infty} \frac{\log U_{k}(b)}{k \log m}, \\
& \underline{\operatorname{dim}}_{B}\left(\bigcup_{z \in \Gamma_{b}}\left[E \cap \Pi_{a, z}\right]\right)=\liminf _{k \rightarrow \infty} \frac{\log U_{k}(b)}{k \log m} .
\end{aligned}
$$

We notice that

$$
U_{k}(b) \geq V_{k}(b) \geq s^{k}
$$

On the other hand, it suffices to verify

$$
U_{k}(b) \leq m^{n} N_{k}(b) \leq m^{n}\left(\# \Gamma_{m^{k} b}\right) s^{k} \leq m^{n}\left(A^{+}-A^{-}+1\right) s^{k} .
$$

In fact, by (3.9) and (3.10), we have

$$
\operatorname{dim}_{B}\left(\bigcup_{z \in \Gamma_{b}}\left[E \cap \Pi_{a, z}\right]\right)=\lim _{k \rightarrow \infty} \frac{\log U_{k}(b)}{k \log m}=\frac{\log s}{\log m} .
$$

To verify (3.10), given an $m$-adic cube of side length $m^{-k}$ intersecting $E \cap \Pi_{a, z}$, denote by $B$, then $B \cap E \cap \Pi_{a, z} \neq \emptyset$, i.e.,

$$
B \cap\left(\bigcup_{v_{1} \cdots v_{k} \in \Omega^{k}} f_{v_{1} \cdots v_{k}}(E)\right) \cap \Pi_{a, z} \neq \emptyset .
$$

Then for some $v_{1} \cdots v_{k} \in \Omega^{k}$,

$$
\begin{aligned}
& B \cap f_{v_{1} \cdots v_{k}}(E) \cap \Pi_{a, z} \neq \emptyset \\
\Longrightarrow & B \cap f_{v_{1} \cdots v_{k}}(E) \neq \emptyset \text { and } f_{v_{1} \cdots v_{k}}(E) \cap \Pi_{a, z} \neq \emptyset \\
\Longrightarrow & B \cap f_{v_{1} \cdots v_{k}}\left([0,1]^{n}\right) \neq \emptyset \text { and } f_{v_{1} \cdots v_{k}}\left([0,1]^{n}\right) \cap \Pi_{a, z} \neq \emptyset,
\end{aligned}
$$

which implies

Hence (3.10) holds.

$$
U_{k}(b) \leq m^{n} N_{k}(b)
$$

Using Lemma 4 and the above proposition, we have

Proposition 3. For all $b \in \mathbf{R}$,

$$
\operatorname{dim}_{B}\left(F_{a, b}\right)=\frac{\log s}{\log m}=\operatorname{dim}_{H} E-1 .
$$


Then Theorem 1(1) follows from Proportions 2 and 3.

\section{Proof of Theorem 1(2)}

In this section, we will show that the Hausdorff dimension of the slice equals its box dimension almost everywhere. During the proof we will use the following result provided by Ledrappier (see [5] Proposition 2.6).

Lemma 9. (Ledrappier) Let $T_{m}$ denote the endomorphism $T_{m} x=m x(\bmod 1)$ of the $(n-1)$-dimensional torus $\mathbf{T}^{n-1}$, and let $S$ be a continuous transformation of a metric space $Y$. Assume that $\Lambda \subset \mathbf{T}^{n-1} \times Y$ is compact and invariant under the map $T_{m} \times S$, and that $\nu$ is an $S$-invariant probability measure on $Y$. Then for $\nu$-a.e. $y$, we have

$$
\operatorname{dim}_{H}\left[\pi^{-1}(y)\right]=\operatorname{dim}_{B}\left[\pi^{-1}(y)\right],
$$

where $\pi: \Lambda \rightarrow Y$ is the projection onto the second coordinate.

Recall that $\tau: \mathbf{T}^{n} \rightarrow \mathbf{T}^{n}$ is the endomorphism

$$
\tau(y)=m y(\bmod 1) .
$$

Proof of Theorem 1(2). Suppose $a=\left(a_{1}, \cdots, a_{n}\right) \in \mathbf{Z}^{n} \backslash\{0\}$, without loss of generality, we may assume

$$
a_{n} \neq 0
$$

For any fixed $b \in \mathbf{R}$, we have

$$
F_{a, b}=F \cap\left\{x=\left(x_{1}, \cdots, x_{n}\right) \in \mathbf{T}^{n}: a \cdot x \equiv b(\bmod 1)\right\} .
$$

Let $T_{m}$ denote the endomorphism $T_{m} x=m x(\bmod 1)$ of the $(n-1)$-dimensional torus $\mathbf{T}^{n-1}, S(x)=m x(\bmod 1)$ the map on one-dimensional torus $\mathbf{T}$, and $g: \mathbf{T}^{n} \rightarrow$ $\mathbf{T}^{n}$ the map

$$
g(x)=\left(x_{1}, \cdots, x_{n-1}, a \cdot x(\bmod 1)\right) .
$$

Then

$$
\tau=T_{m} \times S
$$

and both $F$ and $g(F)$ are $\tau$-invariant, i.e., $F=\tau(F)$ and

$$
\tau(g(F))=g(\tau(F))=g(F)
$$

since $g \circ \tau=\tau \circ g$.

Since $\left|a_{n}\right| \geq 1$, for $i=0, \cdots,\left(3\left|a_{n}\right|-1\right)$, we let

$$
B_{i}=\left\{y \in\left[\frac{i}{3\left|a_{n}\right|}, \frac{i+1}{3\left|a_{n}\right|}\right](\bmod 1)\right\} \subset \mathbf{T} .
$$

Let

$$
h(x)=a_{n} x
$$

which is defined on the torus $\mathbf{T}$. Then $\left.h\right|_{B_{i}}$ is a bi-Lipschitz map. Set $a^{\prime}=$ $\left(a_{1}, \cdots, a_{n-1}\right)$, we have

$$
g(x, y)=\left(x, a^{\prime} \cdot x+a_{n} y\right) \text { for all } x \in \mathbf{T}^{n-1} \text { and } y \in \mathbf{T} .
$$

It is easy to check that

$$
\left.g\right|_{\mathbf{T}^{n-1} \times B_{i}} \text { is a bi-Lipschitz endomorphism. }
$$

Since $\mathbf{T}^{n}=\cup_{i}\left(\mathbf{T}^{n-1} \times B_{i}\right)$, by (4.1), we obtain that

$$
\operatorname{dim} g\left(F_{a, b}\right)=\operatorname{dim} F_{a, b}
$$


where 'dim' stands any one of $\operatorname{dim}_{H}, \underline{\operatorname{dim}}_{B}$ and $\overline{\operatorname{dim}}_{B}$.

Now, let $Y=\mathbf{T}$ equipped with a normalized Lebesgue measure $\nu$. Since

$$
\pi^{-1}[b(\bmod 1)]=g\left(F_{a, b}\right),
$$

then by the previous lemma, for $\nu$-almost all $b \in \mathbf{T}$,

$$
\operatorname{dim}_{H} g\left(F_{a, b}\right)=\operatorname{dim}_{B} g\left(F_{a, b}\right) .
$$

Therefore, it follows from (4.2) that for $\mathcal{L}$-almost all $b \in \mathbf{R}$,

$$
\operatorname{dim}_{H} F_{a, b}=\operatorname{dim}_{B} F_{a, b} .
$$

This completes the proof of Theorem 1(2).

\section{Proof of Theorem 2}

Recall that

$$
\Lambda=\left\{b \in\left[A^{-}, A^{+}\right]: E \cap \Pi_{a, b} \neq \emptyset\right\} .
$$

In this section, we will give the Hausdorff and box dimension of $E_{a, b}$ for $\mathcal{L}$-almost all $b \in \Lambda$.

The following lemma is useful (for details, see [10] and [11]).

Lemma 10. The following functions

$$
g_{1}(b)=\operatorname{dim}_{H}\left[E \cap \Pi_{a, b}\right], \quad g_{2}(b)=\underline{\operatorname{dim}}_{B}\left[E \cap \Pi_{a, b}\right] \text { and } g_{3}(b)=\overline{\operatorname{dim}}_{B}\left[E \cap \Pi_{a, b}\right]
$$

are Borel measurable.

We will show some properties about $\Lambda$.

Proposition 4. $\Lambda$ is a self-similar set with positive Lebesgue measure, i.e.,

$$
\Lambda=\bigcup_{v \in \Omega} S_{v}(\Lambda) \text { and } \mathcal{L}(\Lambda) \geq 1
$$

Further,

$$
\mathcal{L}\left(\left\{b \in \Lambda: \operatorname{dim}_{H} E_{a, b}=\frac{\log s}{\log m}\right\}\right) \geq 1 .
$$

Proof. First, we will show that $\Lambda$ is compact. In fact, we only need to show that $\Lambda$ is closed, since $\Lambda \subset\left[A^{-}, A^{+}\right]$is bounded. For any $b \in \Lambda^{c}, E \cap \Pi_{a, b}=\emptyset$, which implies for sufficiently small $\varepsilon>0$,

$$
E \cap\left\{x \in \mathbf{R}^{n}: a \cdot x \in(b-\varepsilon, b+\varepsilon)\right\}=\emptyset .
$$

That means $\Lambda^{c}$ is open. Hence $\Lambda$ is closed and thus compact.

By (3.1) in Lemma 5, we know that

$$
E \cap \Pi_{a, b}=\bigcup_{v \in \Omega} f_{v}\left(E \cap \Pi_{a, T_{v}(b)}\right) .
$$

Then for any $b \in \Lambda$, the above formula implies

$$
\begin{aligned}
E \cap \Pi_{a, b} \neq \emptyset & \Longleftrightarrow \exists v \in \Omega \text {, s.t. } E \cap \Pi_{a, T_{v}(b)} \neq \emptyset \\
& \Longleftrightarrow \exists v \in \Omega \text {, s.t. } T_{v}(b) \in \Lambda \\
& \Longleftrightarrow \exists v \in \Omega \text {, s.t. } b \in S_{v}(\Lambda),
\end{aligned}
$$

which means

$$
\Lambda=\bigcup_{v \in \Omega} S_{v}(\Lambda)
$$


Therefore $\Lambda$ is a self-similar set.

Now we will show that

$$
\mathcal{L}(\Lambda) \geq 1
$$

By Theorem 1(2), we know that

$$
\max _{z \in \Gamma_{b}}\left(\operatorname{dim}_{H} E_{a, b}\right)=\operatorname{dim}_{H} \bigcup_{z \in \Gamma_{b}} E_{a, b}=\frac{\log s}{\log m} \text { for } \mathcal{L} \text {-a.e. } b \in\left[A^{-}, A^{+}\right] .
$$

Hence for $\mathcal{L}$-a.e. $b \in\left[A^{-}, A^{+}\right]$,

$$
\exists b^{\prime} \in\left[A^{-}, A^{+}\right] \cap(b+\mathbf{Z})\left(=\Gamma_{b}\right) \text { s.t. } \operatorname{dim}_{H} E_{a, b^{\prime}}=\frac{\log s}{\log m} .
$$

Let

$$
K:=\left\{b \in \Lambda: \operatorname{dim}_{H} E_{a, b}=\frac{\log s}{\log m}\right\} .
$$

Then $K$ is Borel measurable from Lemma 10. We have $K \subset \Lambda$ and

$$
\mathcal{L}(K)=\mathcal{L}\left(\bigcup_{i=1}^{A^{+}-A^{-}}\left(K \cap I_{i}\right)\right)=\sum_{i=1}^{A^{+}-A^{-}} \mathcal{L}\left(K \cap I_{i}\right)
$$

It follows from (5.3) that

$$
b \in \bigcup_{i=1}^{\left(A^{+}-A^{-}\right)} \bigcup_{j=-i+1}^{\left(A^{+}-A^{-}-i\right)}\left(K \cap I_{i}+j\right) \text { for } \mathcal{L} \text {-a.e. } b \in\left[A^{-}, A^{+}\right] .
$$

By the above formula, we obtain that

$$
\begin{aligned}
\left(A^{+}-A^{-}\right) & =\mathcal{L}\left(\left[A^{-}, A^{+}\right]\right) \leq \sum_{i=1}^{\left(A^{+}-A^{-}\right)} \sum_{j=-i+1}^{\left(A^{+}-A^{-}-i\right)} \mathcal{L}\left(K \cap I_{i}+j\right) \\
& \leq \sum_{i=1}^{A^{+}-A^{-}}\left(A^{+}-A^{-}\right) \mathcal{L}\left(K \cap I_{i}\right) \leq\left(A^{+}-A^{-}\right) \mathcal{L}(K)
\end{aligned}
$$

which implies

$$
\mathcal{L}(\Lambda) \geq \mathcal{L}(K) \geq 1
$$

This completes the proof.

We will also use the following key technique in [11].

Lemma 11. [11] If $B \subset \Lambda$ is a Borel measurable set such that

$$
\bigcup_{v \in \Omega} S_{v}(B) \subset B
$$

then $\mathcal{L}(B)=\mathcal{L}(\Lambda)$ or 0 .

Now we will give the proof of Theorem 2 .

Proof of Theorem 2. Let $K_{1}=\left\{b \in \Lambda: g_{1}(b)=\operatorname{dim}_{H} E_{a, b} \geq \frac{\log s}{\log m}\right\}$. Then $K_{1}$ is Borel measurable with $\mathcal{L}\left(K_{1}\right) \geq \mathcal{L}(K) \geq 1$ due to Lemma 10 and (5.2).

According to Lemma 5 , for any $v \in \Omega$ and $b \in K_{1}$,

$$
E \cap \Pi_{a, S_{v}(b)} \supset \frac{E \cap \Pi_{a, b}+v}{m}=f_{v}\left(E \cap \Pi_{a, b}\right),
$$


then

$$
\operatorname{dim}_{H}\left(E \cap \Pi_{a, S_{v}(b)}\right) \geq \operatorname{dim}_{H}\left(f_{v}\left(E \cap \Pi_{a, b}\right)\right)=\operatorname{dim}_{H}\left(E \cap \Pi_{a, b}\right) \geq \frac{\log s}{\log m},
$$

which implies $S_{v}(b) \in K_{1}$. Hence $S_{v}\left(K_{1}\right) \subset K_{1}$ for any $v \in \Omega$, i.e.,

$$
\bigcup_{v \in \Omega} S_{v}\left(K_{1}\right) \subset K_{1}
$$

Notice that $K_{1} \subset \Lambda$. Then it follows from Lemma 11 that

$$
\mathcal{L}\left(K_{1}\right)=\mathcal{L}(\Lambda)
$$

That means

Whereas for all $b \in \mathbf{R}$,

$$
\operatorname{dim}_{H} E_{a, b} \geq \frac{\log s}{\log m} \text { for } \mathcal{L} \text {-a.e. } b \in \Lambda .
$$

$$
\operatorname{dim}_{H} E_{a, b} \leq \underline{\operatorname{dim}}_{B} E_{a, b} \leq \overline{\operatorname{dim}}_{B} E_{a, b} \leq \operatorname{dim}_{B}\left(\bigcup_{z \in \Gamma_{b}} E_{a, z}\right)=\frac{\log s}{\log m},
$$

hence

$$
\operatorname{dim}_{H} E_{a, b}=\operatorname{dim}_{B} E_{a, b}=\frac{\log s}{\log m} \text { for } \mathcal{L} \text {-a.e. } b \in \Lambda \text {. }
$$

\section{References}

[1] BÁrÁny, B., A. Ferguson, and K. Simon: Slicing the Sierpinski gasket. - Nonlinearity 25, 2012, 1753-1770.

[2] Benjamini, I., and Y. Peres: On the Hausdorff dimension of fibres. - Israel J. Math. 74, 1991, 267-279.

[3] Hawkes, J.: Some algebraic properties of small sets. - Q. J. Math. 26, 1975, 195-201.

[4] Hutchinson, J. E.: Fractals and self similarity. - Indiana Univ. Math. J. 30, 1981, 713-747.

[5] Kenyon, R., and Y. Peres: Intersecting random translates of invariant Cantor sets. - Invent. Math. 104, 1991, 601-629.

[6] LiU, Q. H., L. F. Xi, and Y. F. ZhaO: Dimensions of intersections of the Sierpinski carpet with lines of rational slopes. - Proc. Edinb. Math. Soc. 50, 2007, 411-428.

[7] Manning, A., and K. Simon: Dimension of slices through the Sierpinski carpet. - Trans. Amer. Math. Soc. 365, 2013, 213-250.

[8] Marstrand, J. M.: Some fundamental geometrical properties of plane sets of fractional dimension. - Proc. London Math. Soc. 4, 1954, 257-302.

[9] Mattila, P.: Geometry of sets and measures in Euclidean spaces. - Cambridge Univ. Press, 1995 .

[10] Mattila, P., and R. D. Mauldin: Measure and dimension functions: measurability and densities. - Math. Proc. Cambridge Philos. Soc. 121:1, 1997, 81-100.

[11] Wen, Z. Y., and L. F. XI: On the dimensions of sections for the graph-directed sets. - Ann. Acad. Sci. Fenn. Math. 35, 2010, 515-535.

[12] Wu, W., and L. F. XI: Dimensions of slices through a class of generalizd Sierpinski sponges. - J. Math. Anal. Appl. 399, 2013, 514-523. 